\title{
Accounting for the Variation of Driver Aggression in the Simulation of Conventional and Advanced Vehicles
}

\author{
Jeremy S. Neubauer and Eric Wood \\ National Renewable Energy Laboratory
}

doi: $10.4271 / 2013-01-1453$

\section{ABSTRACT}

Hybrid electric vehicles, plug-in hybrid electric vehicles, and battery electric vehicles offer the potential to reduce both oil imports and greenhouse gases, as well as to offer a financial benefit to the driver. However, assessing these potential benefits is complicated by several factors, including the driving habits of the operator. We focus on driver aggression, i.e., the level of acceleration and velocity characteristic of travel, to (1) assess its variation within large, real-world drive datasets, (2) quantify its effect on both vehicle efficiency and economics for multiple vehicle types, (3) compare these results to those of standard drive cycles commonly used in the industry, and (4) create a representative drive cycle for future analyses where standard drive cycles are lacking.

\section{INTRODUCTION}

Hybrid electric vehicles (HEVs), plug-in hybrid electric vehicles (PHEVs), and battery electric vehicles (BEVs) offer the potential to reduce both oil imports and greenhouse gases, as well as to offer a financial benefit to the driver. However, assessing these potential benefits is complicated by several factors, including the local climate, the cleanliness of the grid supplying electricity (for PHEVs and BEVs), and the driving habits of the operator, among many other things. Driving habits can be divided into two topics for consideration: (1) trip patterns, i.e., the distribution of trip times and distances, and (2) aggression, i.e., the level of acceleration and the velocity characteristics of travel. Investigation of trip patterns in [1] and [2] found that the variation in the distribution of daily miles traveled observed in real-world, multi-day drive data [ $\underline{3}]$ produces significant variation in gasoline savings and total cost of ownership.
Herein, we focus on driver aggression to (1) assess its variation within large, real-world drive datasets, (2) quantify its effect on both vehicle efficiency and economics for multiple vehicle types, (3) compare these results to those of standard drive cycles commonly used in the industry, and (4) create a representative drive cycle for future analyses where standard drive cycles are lacking. By doing so, we aim to supply an approach for vehicle performance simulation and testing that accurately captures the variation between different drivers, in particular for high-level techno-economic analyses performed using the National Renewable Energy Laboratory's (NREL's) Battery Ownership Model. This work is supported by the U.S. Department of Energy's Vehicle Technologies Program.

\begin{abstract}
$\underline{\text { ANALYSIS }}$
In this study, we apply a high-resolution vehicle simulator to real-world drive data to calculate a distribution of vehicle efficiencies for multiple vehicle types and operational modes. We then analyze these distributions and compare them to standard drive cycles commonly employed in the industry. From there, we synthesize and validate an artificial drive cycle that characterizes the average of the complete set of real-world drive data. We also describe in detail a set of associated scaling factors valid for computing the observed variation in vehicle efficiency.
\end{abstract}

\section{Real-World Drive Data}

Real-world, high-accuracy, and high-resolution vehicular velocity histories are needed to predict the actual on-road variation in vehicle efficiencies of different driver and powertrain combinations. For this purpose, 2,154 unique vehicle records (spanning 1-2 days each) were sourced from the NREL Secure Transportation Data Center: a composite of data from Los Angeles, CA; Austin, TX; San Antonio, TX; 
and Houston, TX travel studies [4]. The data were recorded using on-board global positioning system data acquisition systems filtered down to second-by-second acceleration and velocity histories per the methods described in []].

The box charts in Figures $1, \underline{2}, \underline{3}$ show a statistical summary of this composite vehicle record data set broken out by city for vehicular speed, acceleration, and kinetic intensity [6]. Note that both speed and acceleration are averaged over time for each individual vehicle record. For reference, these same statistics are included for four industry-standard drive cycles: (1) Highway Fuel Economy Driving Schedule (HWFET), (2) Urban Dynamometer Driving Schedule (UDDS), (3) Air Resources Board Dynamometer Driving Schedule (LA92), and (4) Supplemental Federal Test Procedure (US06). The median, $25^{\text {th }}$ percentile, and $75^{\text {th }}$ percentile values for speed and kinetic intensity are fairly consistent among all four cities. Acceleration statistics are visibly larger for Los Angeles, but they are consistent for the three Texas cities.

\section{Vehicle Simulation}

Vehicle simulation is conducted to achieve two goals: (1) to generate powertrain specifications for models of comparable conventional vehicles (CVs), HEVs, PHEVs, and BEVs; and (2) to simulate the fuel consumption of these different powertrains when subjected to the driving requirements of real-world operators (as defined by the real-world drive data discussed previously).

For both tasks, we employ the NREL-developed ADVISOR vehicle simulator to calculate the energy consumption of different powertrains under both industry-standard and realworld drive cycles [7]. Operating in the MATLAB/Simulink environment, ADVISOR employs a hybrid backward/ forward-facing approach to evaluate system interactions and performance relative to individual component limitations.

To calculate the powertrain specifications of comparable CVs, HEVs, PHEVs, and BEVs, we simulate each architecture iteratively with different combustion engines, electric motors, and battery sizes until a 0-60 mph acceleration time of 9 seconds and a 40- or 75-mile allelectric range (AER) for the PHEV and BEV, respectively, are achieved simultaneously. Note that the range is determined via the usable energy of the battery and the calculated vehicle efficiency of the UDDS and HWFET cycles combined and adjusted in a manner representative of the two-cycle approximation to the U.S. Environmental Protection Agency's combined city and highway windowsticker rating per [ $[\mathbf{8}$. A midsize sedan with a coefficient of drag of 0.3 and a frontal area of $2.27 \mathrm{~m}^{2}$ is assumed in all cases, as is a $136 \mathrm{~kg}$ cargo mass. The HEV and PHEV are modeled with a parallel engine/motor configuration, and are held to a $40 \%$ degree of hybridization. The HEV battery was sized to approximate existing commercial HEVs [9]. Additional inputs and results are presented in Table 1.

Subsequently, we apply these models to calculate the vehicle efficiency of each powertrain for four industry-standard drive cycles (HWFET, UDDS, LA92, and US06), as well as each of the processed real-world drive days discussed above. For vehicle record simulations, we lock the PHEV in charge depleting (CD) and charge sustaining (CS) modes separately; in doing so, we ignore the AER limitations of the PHEV and allow it to operate in $\mathrm{CD}$ mode indefinitely.

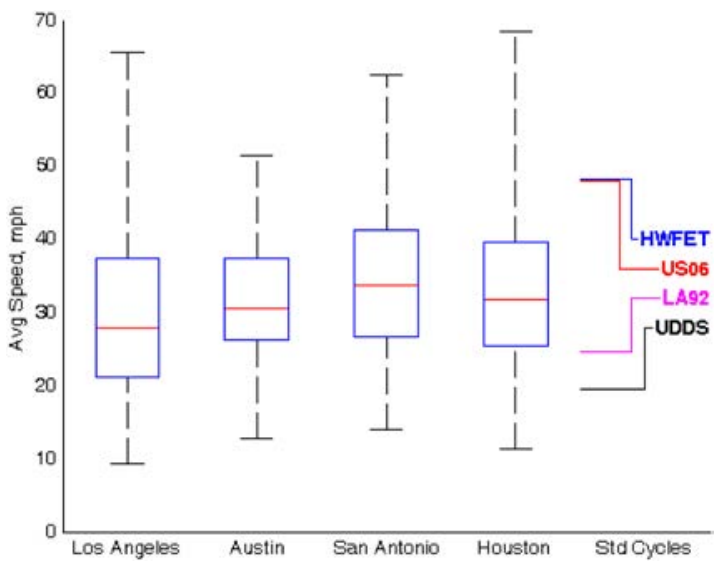

Figure 1. Speed statistics for four real-world drive data sets

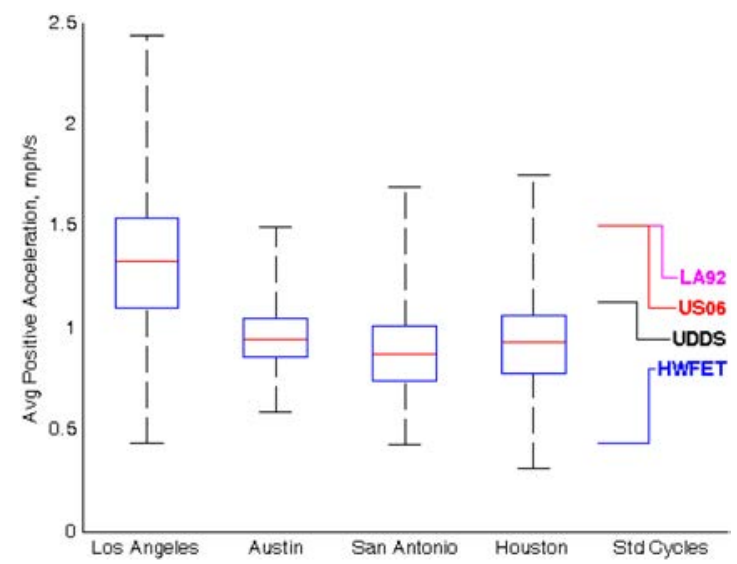

Figure 2. Acceleration statistics for four real-world drive data sets

We approach the BEV the same by ignoring AER limitations. This approach is used so that the entire vehicle record may be simulated regardless of distance travelled. We further assume that initial engine coolant is at operational temperature at the beginning of each trip. We recognize that such assumptions are not realistic of actual vehicle use, but they are important to ensure the impact of local climate and that the timing of trips may be separated from kinetic intensity. The effect of AER, climate, and cold starts will be addressed in a future publication as the appropriate level of consideration of multiday driving patterns, thermal effects, and battery/engine 
Table 1. Vehicle Specifications

\begin{tabular}{lcccc}
\hline \hline Powertrain & CV & HEV & PHEV40 & BEV75 \\
\hline Accessory Load (W) & 700 & 300 & 300 & 300 \\
Vehicle Mass (kg) & 1,679 & 1,613 & 1,794 & 1,611 \\
Internal Combustion Engine & 121 & 62 & 69 & $\mathrm{n} / \mathrm{a}$ \\
Power (kW) & $\mathrm{n} / \mathrm{a}$ & 41 & 46 & 95 \\
Electric Motor Power (kW) & $\mathrm{n} / \mathrm{a}$ & 1.67 & 17.6 & 25.5 \\
Battery Total Energy (kWh) & $\mathrm{n} / \mathrm{a}$ & $80 \%$ & $95 \%$ & $95 \%$ \\
Battery Maximum State of & $\mathrm{n} / \mathrm{a}$ & $60 \%$ & $25 \%$ & $5 \%$ \\
Charge & & $38.6 \mathrm{mpg}$ & $34.2 \mathrm{mpg}(\mathrm{CS})$ & $305 \mathrm{Wh} / \mathrm{mi}$ \\
Battery Minimum State of & $27.0 \mathrm{mpg}$ & & & \\
Charge & & & & \\
Vehicle Efficiency & &
\end{tabular}

control strategy is beyond the scope of this paper. The effects of grade, wind, and cornering are also neglected.

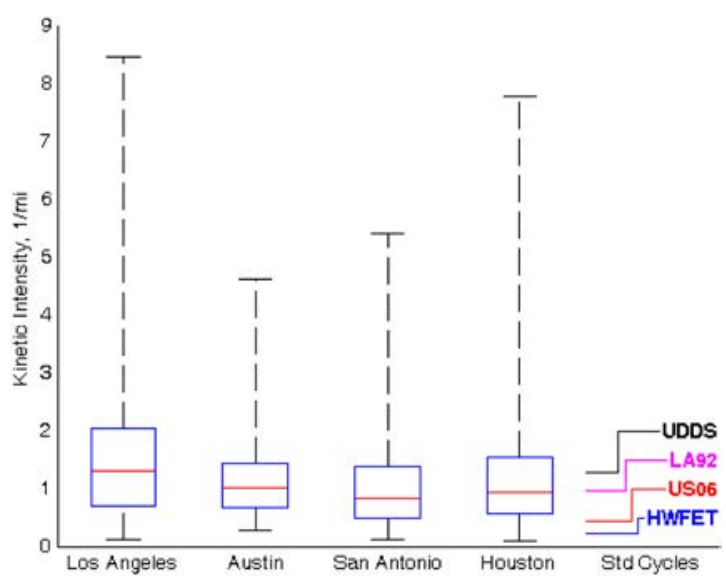

Figure 3. Kinetic intensity statistics for four real-world drive data sets

It is worth noting that the driving data employed herein were collected from an array of vehicle makes, models, years, and body styles from the existing local fleet of personal-use vehicles. The authors acknowledge that some drive data evaluated herein were likely collected from vehicles with greater performance capabilities than the employed models (namely maximum acceleration, where our simulated vehicles are capable of a 9-second $0-60-\mathrm{mph}$ sprint). Accordingly, we checked all simulated trips to evaluate the occurrence of speed trace errors where our simulated vehicle was incapable of matching the original recorded speed history. We found that of the 13,622 real-world trips simulated across five powertrains, $95.9 \%$ of all trips exhibited a maximum error of less than $1 \mathrm{mph}$, while $99.5 \%$ achieved a maximum error of less than $5 \mathrm{mph}$. As the occurrence of speed trace error is small, we conclude that the discrepancies between the performance capability of our simulated vehicle and that of the vehicles behind the data will have a negligible impact on our results. Thus, we do not down-select vehicle records for analysis based on performance criteria.

\section{Effect of Driver and Vehicle}

Variation of vehicle efficiency across individual vehicle records by powertrain (and operational mode, for the PHEV) is presented in Figure 4. Values on the y-axis have been nondimensionalized relative to the average vehicle efficiency for each case, such that values less than one indicate superior efficiency relative to the mean and vice-versa. PHEV efficiency in CD mode is captured by converting any fuel use by the internal combustion engine (occurring in approximately half the data) to an equivalent amount of electrical energy, using the lower heating value for gasoline, and summing with electric motor energy consumption on a per-distance basis. Several important points become clearly evident from this graph. First, we see that the median vehicle efficiency from the data set is relatively close to the mean. Second, the distribution is asymmetric around the meanvariations in aggression have a significantly greater capability to increase fuel consumption from the mean than to decrease it.

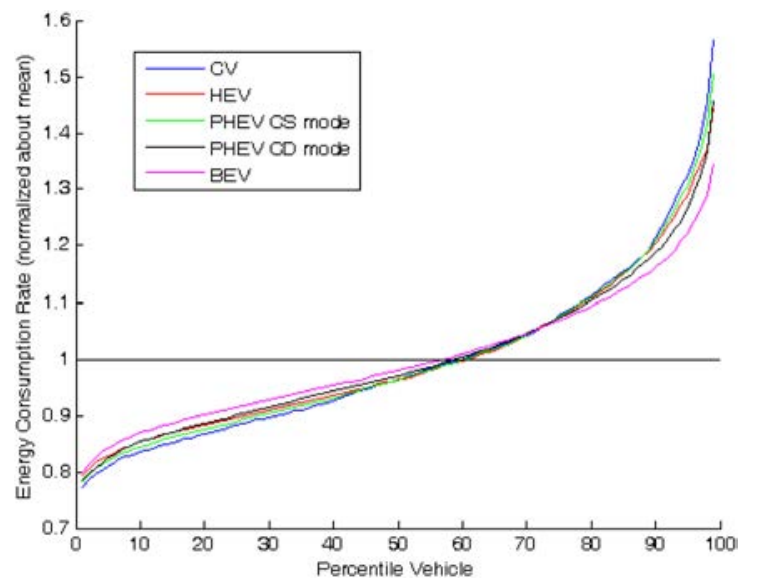

Figure 4. Normalized vehicle efficiency vs. percentile of vehicle records for different powertrains

Finally, we find that the powertrain type has minimal influence on the distribution of vehicle efficiency as a 
function of population percentile. It is important to highlight, though, that this may not be true for different vehicle platforms (e.g., compact cars or large SUVs), those with different $0-60 \mathrm{mph}$ acceleration targets, or hybrids of different architectures (e.g., serial hybrids).

\section{Comparison to Standard Drive Cycles}

Figures $5, \underline{6}, \underline{7}, \underline{8}, \underline{9}$ compare the distribution of vehicle efficiency computed from real-world vehicle records to that calculated for four industry-standard drive cycles (HWFET, UDDS, LA92, and US06) for four vehicle types (CV, HEV, PHEV, and BEV). Two plots are shown for the PHEV, one for $\mathrm{CS}$ operation and one for $\mathrm{CD}$, split for the reasons noted above.

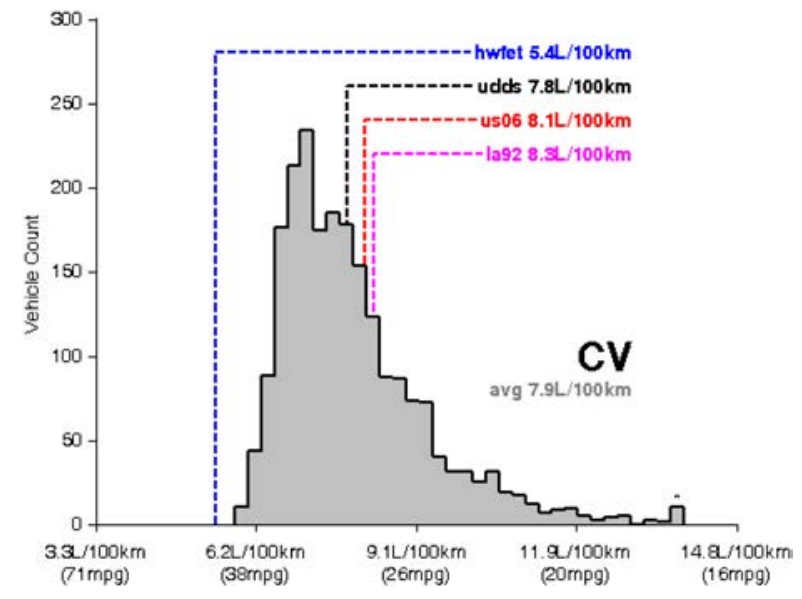

Figure 5. Comparison of vehicle record and standard cycle $C$ V efficiency



Figure 6. Comparison of vehicle record and standard cycle HEV efficiency

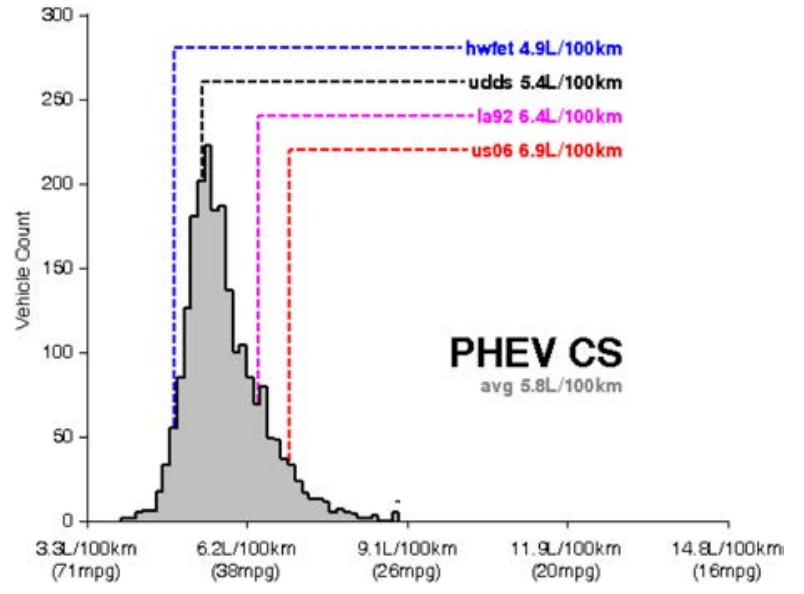

Figure 7. Comparison of vehicle record and standard cycle PHEV efficiency in charge sustaining mode



Figure 8. Comparison of vehicle record and standard cycle PHEV efficiency in charge depleting mode

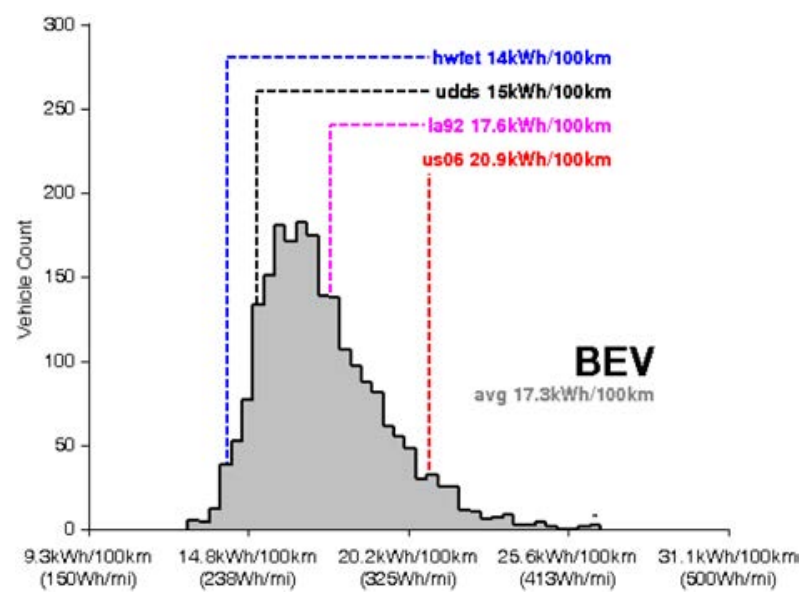

Figure 9. Comparison of vehicle record and standard cycle BEV efficiency

These plots show distinct differences between operational modes (combustion-only, hybrid operation, and electricityonly) with respect to how the standard drive cycles compare 
to the distribution of vehicle record results. When operating only under the power of combustion $(\mathrm{CV})$, we find that simulation of the HWFET cycle significantly underpredicts vehicle record fuel consumption calculations, whereas the UDDS is representative of the mean, and the US06 and LA92 cycles slightly overpredict average vehicle record fuel consumption. For the CV, LA92 predicts the highest fuel consumption, but it is relatively close to that predicted by US06. When operating in hybrid mode (HEV, PHEV-CS), we find that the HWFET still predicts the lowest fuel consumption, but it is now within the tail end of the vehicle record data. The UDDS now slightly underestimates average vehicle record simulations, but it represents reasonably well the mode thereof. Further, the differences between UDDS, LA92, and US06 predictions are more exaggerated than in the combustion-only case, and US06 becomes the least fuelefficient standard drive cycle. These trends continue when advancing to all-electric mode (PHEV-CD, BEV). The UDDS cycle falls further down in relative fuel consumption when compared to the mode value of vehicle record data, and the difference between UDDS, LA92, and US06 predictions increases even further. Here we find that the LA92 cycle is the best indicator of average vehicle record predictions among the four standard cycles we examined.

It is important to note that this latter trend of increasing variation in efficiency predictions between standard drive cycles as the degree of electrification increases is not indicative of increasing sensitivity to real-world drivers. Recall that the results in the previous section showed that the variation in fuel efficiency across real-world drive data is fairly consistent across powertrains (see Figure 3). Rather, these data imply that the response of these powertrains to different drive cycles is varied and complex, as will be discussed below.

\section{Creating a Representative Drive Cycle}

A set of drive cycles representative of specific percentiles of the recorded real-world drive data is desired for future simulation. Doing so will greatly speed computational time by reducing the information of thousands of 24-hour or longer drive cycles to a single $\sim 15$-minute drive cycle that can be quickly simulated. However, the complex interplay of powertrain and drive cycle makes it impractical to create such a set, as discussed below. However, we do find it possible to design one drive cycle indicative of the average of all computed vehicle record efficiencies, which then allows the application of scaling factors from Figure 3 to estimate that of a specific population percentile. Herein, we apply the NRELdeveloped DRIVE [10] software package to synthesize this single drive cycle.

DRIVE employs a deterministic multivariate hierarchical clustering method to generate representative drive cycles from source data. It first concatenates our 2,154 vehicle records into a single "super" cycle, which is characterized for more than 170 drive-cycle metrics. The tool then decomposes the cycle into its component microtrips, which are individually analyzed over the same set of operational metrics. This set of statistics includes well-known metrics such as average driving speed, stops per mile, and zero-speed time as a percentage of cycle operation. Other specialized metrics include kinetic intensity, aerodynamic speed, and characteristic acceleration, which are used to characterize energy consumption [ㅁ]. Afterwards, the individual microtrips are grouped into clusters and ranked based on a set of performance metrics. Upon ranking, the ideal microtrip from each cluster is selected and concatenated to form a representative cycle. This clustering process is iterated over the number of clusters chosen for the data as well as the performance metrics chosen for ranking. It is based on a maximum number of clusters, which is calculated as the product of the desired representative cycle duration, the number of stops per mile for the "super" cycle, and the average speed over the "super" cycle. As a final step in the generation of a representative drive cycle, zero-speed time is either added or removed from the final drive cycle output to match the percentage found in the original data "super" cycle.

The resultant drive pattern is shown in Figure 10. It has an average speed of $30.5 \mathrm{mph}$, an average positive acceleration of $0.960 \mathrm{mph} / \mathrm{s}$, and a kinetic intensity of $0.577 \mathrm{mi}^{-1}$. Simulation of this drive cycle in ADVISOR for all four powertrains shows a good agreement of average predicted vehicle efficiency when compared to that observed from the simulation of all 2,154 vehicles, as shown in Table 2. Accordingly, this drive cycle can be used to quickly compute average vehicle efficiency, which can then be expanded to represent different population percentile vehicle efficiencies using the correction factors of Table 3 (computed from the dataset used to prepare Figure 4).

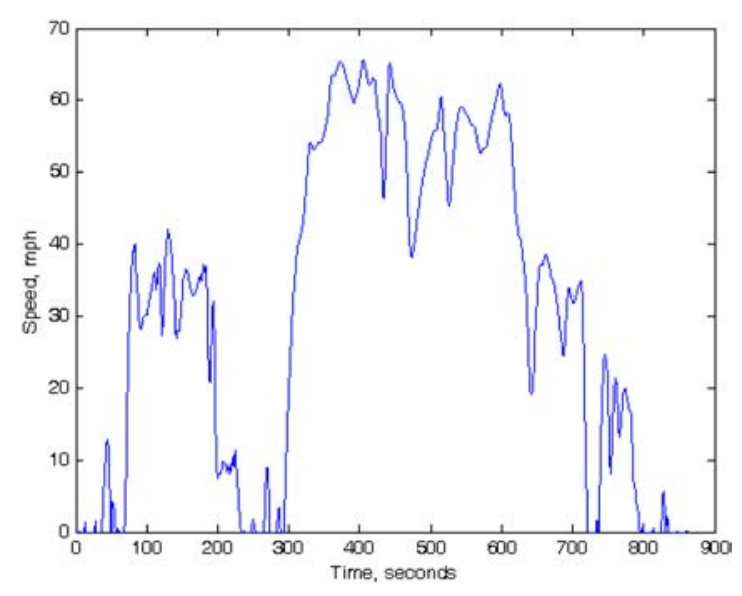

Figure 10. Representative drive cycle produced from 2,154 vehicles using DRIVE 
Table 2. Comparison of Average Predicted Vehicle Efficiency Between Synthesized Drive Cycle and Vehicle Record Simulations

Error of mean vehicle record and synthesized drive-cycle efficiency

\begin{tabular}{ccccc} 
CV & HEV & $\begin{array}{c}\text { PHEV40 } \\
(\text { CS })\end{array}$ & $\begin{array}{c}\text { PHEV40 } \\
(\text { CD })\end{array}$ & BEV75 \\
\hline \hline$-0.37 \%$ & $-0.70 \%$ & $0.07 \%$ & $0.23 \%$ & $-0.03 \%$ \\
\hline
\end{tabular}

Table 3. Scaling Factors

\begin{tabular}{cccccc}
\hline \hline $\begin{array}{c}\text { Vehicle } \\
\text { Day } \\
\text { Percentile }\end{array}$ & CV & HEV & $\begin{array}{c}\text { PHEV40 } \\
\text { (CS) }\end{array}$ & $\begin{array}{c}\text { PHEV40 } \\
\text { (CD) }\end{array}$ & BEV75 \\
\hline \hline $5^{\text {th }}$ & 0.810 & 0.828 & 0.847 & 0.826 & 0.841 \\
$25^{\text {th }}$ & 0.883 & 0.896 & 0.920 & 0.900 & 0.915 \\
$50^{\text {th }}$ & 0.962 & 0.963 & 0.977 & 0.969 & 0.980 \\
$75^{\text {th }}$ & 1.077 & 1.074 & 1.062 & 1.072 & 1.068 \\
$95^{\text {th }}$ & 1.325 & 1.290 & 1.220 & 1.266 & 1.223 \\
\hline
\end{tabular}

\section{Optimal Drive-Cycle Characteristics}

Creation of a suite of drive cycles representative of arbitrary percentile drivers was deemed infeasible. This outcome is the result of individual drivetrains being particularly well-suited to specific drive-cycle characteristics. This point is illustrated in Figure 11, where the relative efficiency of the simulated $\mathrm{CV}$ and HEV are plotted in 4D space. In this plot, the 2,154 vehicle records are segmented into 13,622 trips and plotted with marker $\mathrm{x}-\mathrm{y}$ coordinates relative to cycle speed and acceleration statistics, marker size relative to trip length, and marker color relative to percentile energy consumption rate per the specified powertrain.

In terms of cycle statistics (speed, acceleration, distance), the CV and HEV plots represent the same 13,622 data points. This "point cloud" alone reveals interesting information concerning real-world drive data. We see that low-speed trips (approximately less than $15 \mathrm{mph}$ ) coincide with shortdistance travel, and thus account for relatively small amounts of energy consumption. As travel speed increases, we see a tendency for trip distances to increase while acceleration metrics decrease. The observed inverse relationship between driving speed and acceleration can be linked to both behavioral norms (lack of driver-requested high-acceleration events during high speed travel) and technical realities (inability of most vehicles to deliver high acceleration at high speed).

Concerning relative energy consumption rates, we see that the optimal drive cycle for our CV occupies the intersection of high-speed and low-acceleration travel. The industrystandard HWFET cycle is an exemplar of such a drive cycle, yielding energy consumption rates close to the lower bound. Alternatively, the HEV exhibits optimal energy consumption rates when driven over low speed, low acceleration cycles (near $20 \mathrm{mph}, 0.5 \mathrm{mph} / \mathrm{s}$ ). The differences in gradients between these two maps can be primarily attributed to the effects of regenerative braking made possible through electric hybridization. By recouping energy traditionally lost during braking events, our HEV is not as heavily penalized by the stop-and-go patterns characteristic of low-speed travel. Instead, the HEV achieves its highest fuel consumption values over cycles with high speed/acceleration metrics, presumably as a result of the aerodynamic, rolling, and inertial losses of the classical road load equation.
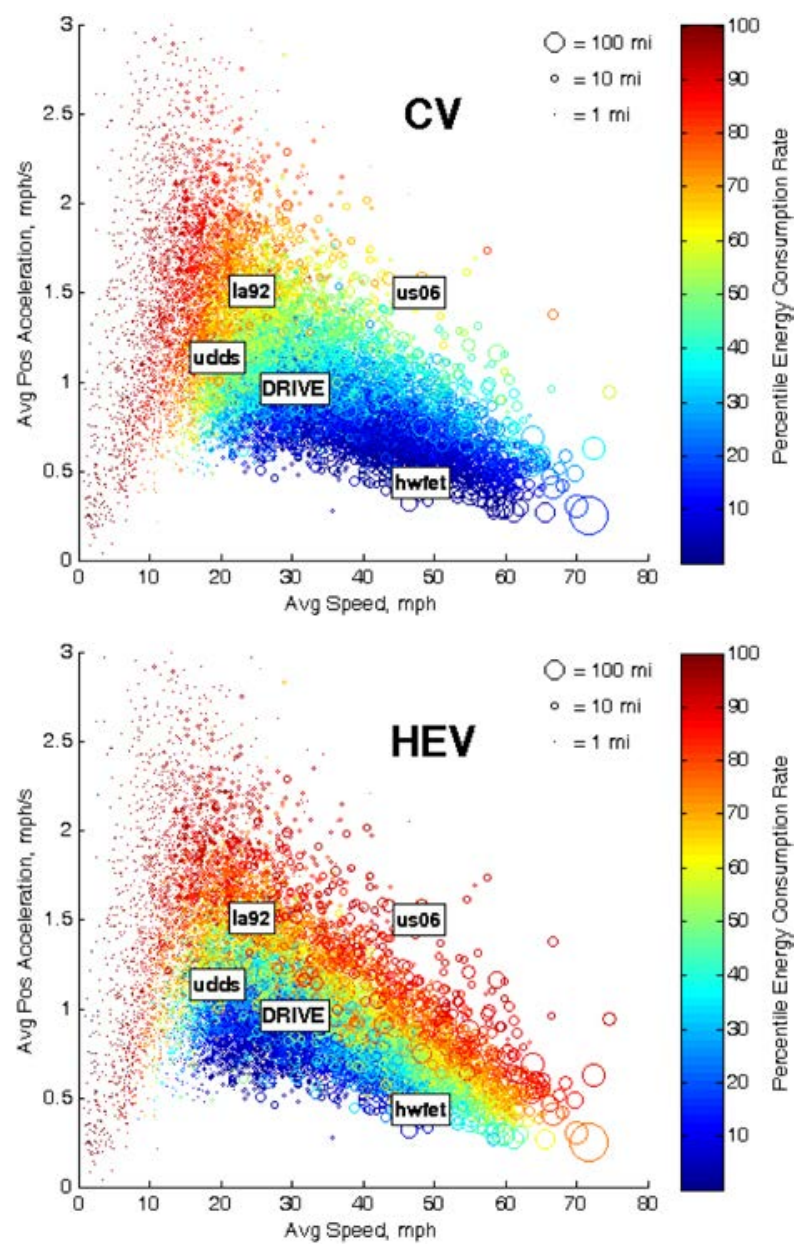

Figure 11. CV and HEV efficiency gradients: marker location indicates the average speed and acceleration of each vehicle record; marker size indicates distance traveled in each vehicle record; and marker color indicates the relative energy consumption rate for each record. The locations of standard test cycles in this $2 D$ space (including the synthesized cycle built using DRIVE) are also indicated. 
These maps illustrate the challenge of synthesizing drive cycles capable of capturing arbitrary percentile energy consumption rates for disparate powertrains and underscore the utility of the synthesized drive cycle (presented in the previous section) in its ability to capture mean energy consumption rate regardless of powertrain configuration.

\section{Relative Efficiency across Powertrains}

Having highlighted the discrepancy between optimal drivecycle characteristics for our $\mathrm{CV}$ and $\mathrm{HEV}$, we now examine the relative efficiency of each drive cycle to understand how vehicle efficiency translates across powertrains.

The data on $\mathrm{CV}$ and HEV efficiency from Figure 11 are repurposed for Figure 12 with percentile HEV fuel rate plotted against percentile CV fuel rate. This representation underscores the discrepancy in optimal drive-cycle characteristics between the simulated $\mathrm{CV}$ and $\mathrm{HEV}$ powertrains. For example, drive cycles falling in the $60^{\text {th }}$ percentile fuel rate when simulated under a $\mathrm{CV}$ powertrain result in HEV fuel rates between the $10^{\text {th }}$ and $90^{\text {th }}$ percentiles.

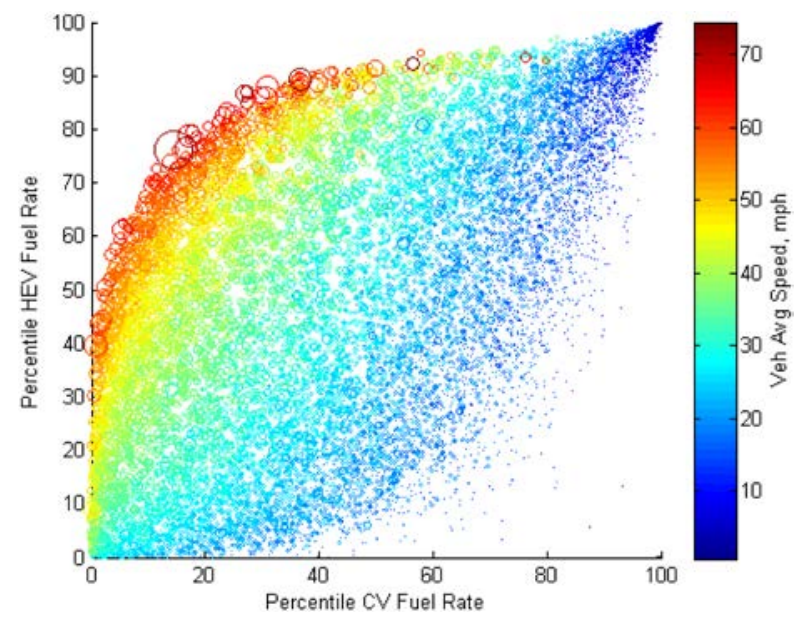

Figure 12. Comparison of $\mathrm{HEV}$ and $\mathrm{CV}$ relative fuel consumption rate for 13,622 simulated drive cycles

Coloring the data by average trip speed provides insight concerning the nature of this discrepancy. This added dimension shows that high-speed trips achieve lower relative vehicle efficiencies in an $\mathrm{HEV}$ when compared to a $\mathrm{CV}$, presumably as a result of the diminishing returns encountered in an HEV in the absence of stop-and-go conditions indicative of low-speed travel. Alternatively, medium-to-low speed trips achieve high relative vehicle efficiency under an $\mathrm{HEV}$ powertrain when compared to a CV. We note, however, that a subset of very low-speed trips exhibits a high relative fuel rate (low relative efficiency). The result is due to a large percentage of vehicle idle time relative to the number of miles traveled.

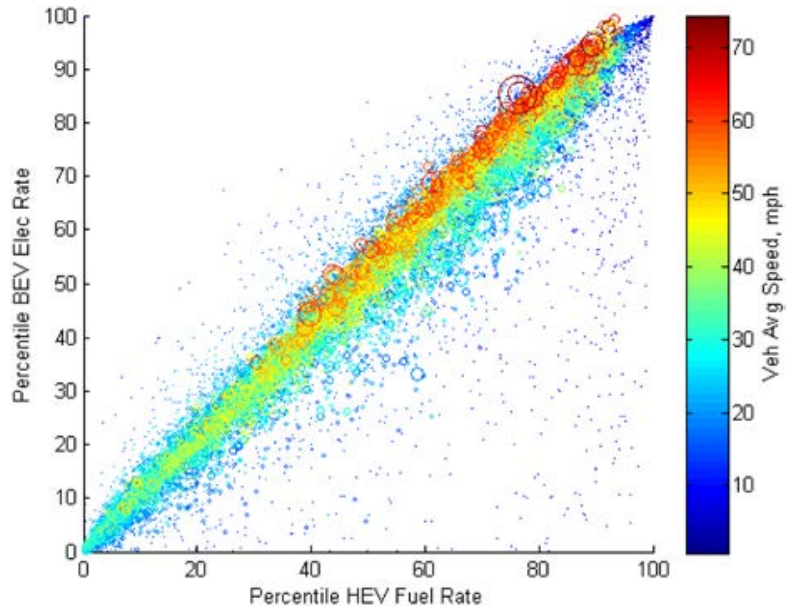

Figure 13. Comparison of $B E V$ and $H E V$ relative fuel consumption rate for 13,622 simulated drive cycles

We take this opportunity to underscore the difference between absolute and relative vehicle efficiency. The HEV powertrain resulted in greater absolute vehicle efficiency than the $\mathrm{CV}$ for each simulated trip. However, specific drive-cycle characteristics are best suited to individual powertrains (e.g., high-speed travel in CVs and low-speed travel in HEVs) resulting in a discontinuity in the comparison of relative efficiency.

Fortunately, this discontinuity in relative efficiency appears unique to comparisons involving the $\mathrm{CV}$. Figure 13 shows a comparison of the HEV and BEV relative efficiencies exhibiting a strong linear relationship between percentile rankings of drive cycles when simulated under distinct powertrains. This linear correlation is representative of the relationship between all combinations of the HEV, PHEV, and $\mathrm{BEV}$ powertrains. The $\mathrm{CV}$ is believed to be the outlier in this analysis due to its lack of regenerative braking, favoring cycles where high average speed is presumably an indicator of infrequent stop-and-go conditions.

\section{CONCLUSIONS}

In this study, we investigated the variation of driver aggression to assess its variation within large, real-world drive datasets, and quantify its effect on vehicle efficiency for multiple vehicle types. We have found that aggression variation between drivers can decrease fuel efficiency by more than $50 \%$ or increase it by more than $20 \%$ from average. Interestingly, across the population we investigated, the normalized efficiency deviation from average as a function of population percentile was found to be largely insensitive to powertrain. However, the specific highefficiency drivers did vary across the CV, HEV, PHEV, and BEV powertrains, implying that ideal driving behavior varies with respect to powertrain. 
Accordingly, the relation of efficiency predicted from realworld drive data to that predicted by the industry-standard HWFET, UDDS, LA92, and US06 drive cycles was not consistent across powertrains, either. Thus, we applied NREL's DRIVE tool to synthesize a drive cycle that is capable of reproducing the average vehicle efficiency predicted from our sampling of real-world drive data. Our results showed that the predicted vehicle efficiency from simulation of this drive cycle agrees with that of simulations of real-world data to within $0.7 \%$ for the four vehicles (five operational modes) addressed herein. Along with the supplied scaling factors, this drive cycle is, therefore, capable of accurately representing the total variation in vehicle efficiency we have observed in the employed vehicle records.

It is important to note that none of the simulated drive cycles account for the effects of grade, wind, large vehicle auxiliary loads (e.g., heating, ventilation, and air-conditioning) or other more complex effects. Accounting for such factors will be the subject of future work.

\section{REFERENCES}

1. Neubauer, J.; Wood, E.; and Brooker, A. "Sensitivity of battery electric vehicle economics to drive patterns, vehicle range, and charge strategies," Journal of Power Sources, 2012; 209, 269-277.

2. Neubauer, J.; Wood, E.; and Brooker, A. "Sensitivity of plug-in hybrid electric vehicle economics to drive patterns, electric range, energy management, and charge strategies," Journal of Power Sources, 2013 (in press).

3. Traffic Choices Study - Summary Report, Puget Sound Regional Council, April 2008.

4. "Secure Transportation Data Project." National Renewable Energy Laboratory. http://www.nrel.gov/ vehiclesandfuels/secure transportation d ata.html [Accessed: August 28, 2012]

5. Duran, A. and Earleywine, M., "GPS Data Filtration Method for Drive Cycle Analysis Applications," SAE Technical Paper 2012-01-0743, 2012, doi:

10.4271/2012-01-0743.

6. O'Keefe, M., Simpson, A., Kelly, K., and Pedersen, D., "Duty Cycle Characterization and Evaluation Towards Heavy Hybrid Vehicle Applications," SAE Technical Paper 2007-01-0302, 2007, doi:10.4271/2007-01-0302.

7. Markel, T.; Brooker, A.; Hendricks, T.; Johnson, V.; Kelly, K.; Kramer, B.; O'Keefe, M.; Sprik, S.; and Wipke, K. "ADVISOR: A Systems Analysis Tool for Advanced Vehicle Modeling," Journal of Power Sources, 2002, 110, pp. 255-266.

8. Revisions and Additions to Motor Vehicle Fuel Economy Label; Final Rule. Washington, D.C.: Federal Register. http:// www.gpo.gov/fdsys/pkg/FR-2011-07- 06/html/

2011-14291.htm [Accessed: Aug 29, 2012]
9. "Hybrid Electric Vehicles." Idaho National Laboratory. http://avt.inl.gov/hev.shtml [Accessed: Sept 5, 2012]

10. "Analysis Tool Generates Custom Vehicle Drive Cycles Based on Real-World Data." National Renewable Energy Laboratory. http://www.nrel.gov/vehiclesandfuels/news/ 2011/1593.html [Accessed: Sept 5, 2012]

\section{ACKNOWLEDGMENTS}

This study was supported by the Energy Storage, Vehicle Technologies Program, Office of Energy Efficiency and Renewable Energy, U.S. Department of Energy. The authors would specifically like to thank Dave Howell and Brian Cunningham of the U.S. Department of Energy for their continued guidance and support. The use of the ADVISOR and DRIVE computational tools, both developed at NREL under funding from the U.S. Department of Energy's Vehicle Technologies Program, was critical to the completion of this study. Special thanks to Adam Duran for supporting our use of DRIVE; Jeff Gonder for supporting the use and analysis of the drive data sources from NREL's Transportation Secure Data Center; and Terry Penney and Ahmad Pesaran for their continual guidance.

\section{DEFINITIONS}

AER - all-electric range

BEV - battery electric vehicle

CD - charge depleting

CS - charge sustaining

CV - conventional vehicle

HEV - hybrid electric vehicle

HWFET - Highway Fuel Economy Driving Schedule

LA92 - Air Resources Board Dynamometer Driving Schedule

NREL - National Renewable Energy Laboratory

PHEV - plug-in hybrid electric vehicle

UDDS - Urban Dynamometer Driving Schedule

US06 - Supplemental Federal Test Procedure 
The Engineering Meetings Board has approved this paper for publication. It has successfully completed SAE's peer review process under the supervision of the session organizer. This process requires a minimum of three (3) reviews by industry experts. ISSN 0148-7191
Positions and opinions advanced in this paper are those of the author(s) and not necessarily those of SAE. The author is solely responsible for the content of the paper.

SAE Customer Service:

Tel: 877-606-7323 (inside USA and Canada)

Tel: 724-776-4970 (outside USA)

Fax: 724-776-0790

Email: CustomerService@sae.org

SAE Web Address: http://www.sae.org

Printed in USA 